\title{
Diagnóstico e tratamento de um caso de odontoma composto-complexo: relato de caso e revisão da literatura
}

\author{
Diagnosis and treatment of a case of complex composite \\ odontoma: case report and literature review
}

\author{
Bruna VAleria TEIXEIRA Merat ${ }^{1}$ \\ LÍDIA SOUZA DE ANDRADE ${ }^{2}$ \\ EUGÊNIO RODRIGUES ARANTES ${ }^{3}$ \\ ANA FLÁVIA SCHUELER dE ASSUMPÇÃo LEITE ${ }^{4}$ \\ SiMONE DE QUEIROZ CHAVES LOURENÇO ${ }^{5}$
}

\begin{abstract}
RESUMO
Objetivo: Apresentar o relato de um caso clínico de um odontoma em maxila anterior e apresentar uma revisão de literatura atualizada do tema. Relato de caso: Paciente com aumento de volume em região palatina de maxila do lado direito, com evolução de aproximadamente dois anos. Após exame clínico, exames radiológicos e hipóteses diagnósticas de lesão intraóssea, foi realizada remoção cirúrgica completa da lesão e análise histopatológica com laudo histopatológico de odontoma composto-complexo. A paciente segue em controle pós-operatório clínico e radiográfico há um ano sem recidiva. Conclusão: Destacase nesse relato a apresentação incomum de um odontoma com características histopatológicas do tipo composto e complexo simultaneamente, achado esse que não alterou o manejo do paciente, nem o prognóstico do caso.
\end{abstract}

Palavras-chaves: Tumores odontogênicos. Anormalidades dentárias. Cirurgia bucal. Odontoma.

\begin{abstract}
Objective: Report the clinical case of an anterior maxilla odontoma and report an updated literature review about the theme. Case report: Pacient with an increase in volume in the palatine region of the right side of the jaw, with an evolution of approximately two years. After clinical examination, radiographs exams and diagnostic hypothesis of intraosseous lesion, it was performed a complete surgical removal of the lesion and histopathological analysis with a histopathological result of compound-complex odontoma. The patient is in clinical and radiographic postoperative one-year control without relapse. Conclusion: It stands out in this report the unusual appearance of an odontoma with complex and compound characteristics simultaneously, without changing the treatment neither the prognosis of the patient.
\end{abstract}

Keywords: Odontogenic tumors. Tooth abnormalities. Oral surgery. Case reports. Odontoma.

Acadêmica de Odontologia, Faculdade de Odontologia da Universidade Federal Fluminense, Niterói, Rio de Janeiro / RJ, Brasil.

2 Residente do Serviço de Cirurgia e Traumatologia Bucomaxilofacial da Universidade Federal Fluminense / Hospital Federal dos Servidores do Estado, Rio de Janeiro / RJ, Brasil.

3 Especialista em Estomatologia pela Universidade Federal do Rio de Janeiro, Rio de Janeiro / RJ, Brasil e em Cirurgia e Traumatologia Bucomaxilofacial pela Universidade Federal Fluminense / Hospital Federal dos Servidores do Estado, Rio de Janeiro / RJ, Brasil.

4 Doutoranda em Odontologia pela Faculdade de Odontologia da Universidade Federal Fluminense, Niterói, Rio de Janeiro / RJ, Brasil.

5 Mestre e Doutora em Patologia Bucal pela Faculdade de Odontologia de Bauru da Universidade de São Paulo, Bauru / SP, Brasil e Professora Associada do Departamento de Patologia da Universidade Federal Fluminense, Niterói / RJ, Brasil. 


\section{INTRODUÇÃO}

Os odontomas, principais tumores odontogênicos, são melhores descritos como distúrbios de desenvolvimento (hamartomas) quandocomparadosàsneoplasiasverdadeiras. ${ }^{1}$ Apresentam etiologia desconhecida, porém, traumas, infecções e fatores genéticos podem surgir como possíveis causas dessa lesão por apresentarem capacidade de levar ao desequilíbrio do processamento genético e do desenvolvimento dentário. ${ }^{2}$ Segundo a Organização Mundial da Saúde (OMS), ${ }^{3}$ os odontomas são classificados em dois grupos, de acordo com a sua diferenciação: complexo e composto.

Radiograficamente, os odontomas complexos apresentam-se como massas amorfas radiopacas, envoltas por uma estreita zona radiolúcida. Otipo composto se assemelha a dentes ou "dentículos" maduros em um único aglomerado. ${ }^{4,5}$ No entanto, a literatura aponta que, ocasionalmente, algumas lesões podem exibir características tanto do complexo quanto do composto. São assintomáticos, de crescimento lento, podendo, em raros casos, chegar a grandes proporções com expansão das corticais ósseas. De modo geral, são descobertos durante exames radiográficos rotineiros ou quando são capazes de provocar um atraso anormal na erupção dentária. ${ }^{1,5,6}$ Sua ocorrência é maior no osso da maxila, onde o tipo composto é mais encontrado, enquanto o tipo complexo é frequentemente mais observado na região de molares de ambos os maxilares. ${ }^{1,4}$

Histologicamente os odontomas são considerados tumores odontogênicos mistos, pois seus tecidos diferenciados são de origem epitelial e mesenquimal. ${ }^{7,8} \mathrm{~A}$ variante histológica e incomum conhecida como odontoma composto-complexo apresenta simultaneamente as características microscópicas do tipo composto e do tipo complexo. Esse padrão combinado se assemelha mais aos hamartomas devido à morfodiferenciação ainda mais desorganizada. Embora seja este o tipo mais comum de tumor odontogênico, a forma composta-complexa é raramente visto na literatura. 9, 10, $11^{-}$

O tratamento através da exérese cirúrgica total é o método mais recomendado na literatura mundial, uma vez que a lesão apresenta um ponto de clivagem que facilita sua remoção completa. Porém, quando essa lesão está associada a um dente irrompido, deve-se, toda vez que possível, preservar o elemento dentário envolvido. ${ }^{1,2} \mathrm{O}$ tratamento cirúrgico conservador apresenta prognóstico favorável, com casos de recidiva considerados raros, além de permitir uma reparação óssea adequada. ${ }^{1,6}$

O propósito deste trabalho é apresentar um caso clínico de alteração óssea de maxila que demonstrou, durante análise histopatológica, características de odontoma compostocomplexo simultaneamente e apresentar revisão de literatura sobre o tema.

\section{Revisão da literatura}

Um dos primeiros relatos do odontoma sendo considerado como uma má formação de desenvolvimento, ou "hamartoma", data de 1943, com Rosoff, ${ }^{12}$ o qual descreve que a composição dos seus tecidos era semelhante aos tecidos que o circundavam, porém, o crescimento desta estrutura se dava de forma desorganizada e sem um padrão específico de desenvolvimento. ${ }^{13}$ Sua etiologia, apesar de ainda desconhecida, pode ser considerada múltipla e estudos como os de Azhar, Kota e El-Nadgy ${ }^{14}$ em 2013 e o de Veis ${ }^{15}$ em 2000 confirmam que a variedade de fatores como traumas, histórico familiar e mutações genéticas podem estar relacionados.

Em 1869, Broca ${ }^{16}$ usou o termo "odontoma" para qualquer tumor originado dos tecidos formativos dentários e sugeriu classificá-los de acordo com o estágio de desenvolvimento 
do dente quando o crescimento anormal começou. ${ }^{16}$ Em uma classificação proposta em 1914, estes foram divididos em três grupos: 1) odontomas epiteliais incluindo neoplasias (que eram conhecidas como cistos multiloculares), 2) odontomas compostos que compreendiam lesões com tecidos anormais derivados do epitélio e do mesênquima com massas calcificadas irregulares parecidas com estruturas de dentes e 3) odontomas do tecido conjuntivo que incluíam outros tumores fibrosos e do tecido conjuntivo com origem apenas mesenquimal. Já Sedano e Pindborg ${ }^{1}$ apresentam a sua classificação de acordo com as suas características morfológicas, dividindoos em composto e complexo.

Quanto às suas características clínicas, a Organização Mundial da Saúde relata que a idade média do diagnóstico pode ocorrer durante as duas primeiras décadas de vida. ${ }^{3}$ Eles podem variar de acordo com a sua área de predileção e aparecem com maior frequência em região maxilar. ${ }^{4} \mathrm{Em}$ 2010, um estudo realizado por Santos, Silva, Florêncio e Da Silva ${ }^{5}$ identificou que o canino incluso é o dente mais afetado no tipo composto, sendo este uma das causas mais comuns para a retenção deste elemento dentário. .,4,5 $^{3}$

Apesar de ambos apresentarem aspectos radiográficos distintos, os odontomas em geral são bem característicos radiograficamente, o que facilita a hipótese diagnóstica antes mesmo de procedimentos cirúrgicos ou análise histopatológica. Principalmente no caso do tipo composto, em que é possível identificar vários dentículos aglomerados de forma e tamanho variáveis, envolvidos numa zona radiotransparente. ${ }^{5}$ Castellon, Valenzuela e Lira em $2000^{17}$ destacaram a importância no estabelecimento do diagnóstico diferencial com outras lesões que apresentam características radiográficas semelhantes como osteomielite esclerosante focal, osteoma, displasia cementária periapical, fibroma ossificante e cementoblastoma. . $^{5} 17$
O tratamento preconizado pela maioria dos trabalhos clínicos da literatura é a excisão cirúrgica, realizada de maneira simples devido à área de clivagem criada entre a lesão intraóssea e o osso através de uma cápsula fibrosa de conjuntivo. ${ }^{1,2,6}$

Apesar da literatura apresentar diversas características que separam as duas variantes no tipo composto e complexo, em casos raros são encontradas as características de ambos os tipos juntos, quando foi descrito como odontoma composto-complexo. ${ }^{11}$

Em 1985, Graydon ${ }^{10}$ relatou em seu estudo um caso de uma paciente de 12 anos de idade que possuía atraso na erupção dos molares superiores. Ao avaliar o exame de imagem, foi observado uma lesão radiopaca bem definida na região do molar superior esquerdo que se estendia do segundo prémolar até a tuberosidade e, superiormente, até o assoalho do seio maxilar. Segundo o autor, foi identificada uma estrutura dentária dentro da massa da lesão com pequenas áreas radiopacas irregulares ao redor. Após a remoção cirúrgica, Graydon ${ }^{10}$ pôde observar que o material coletado apresentava elementos dos tipos composto e complexo. Nóia, ${ }^{4}$ em 2008 , relatou o caso semelhante de uma paciente também do sexo feminino, na segunda década de vida, que apresentava ausência do elemento 33 e persistência do elemento decíduo 73. Foi observada a presença de uma massa radiopaca envolta por um halo radiolúcido, inicialmente sugestivo do tipo complexo. Durante a remoção cirúrgica, contudo, foi evidenciada a presença de dezenas de dentículos e massas amorfas, caracterizando, de acordo com Nóia ${ }^{4}$, um odontoma composto-complexo. ${ }^{4}$

Recentemente, Torul ${ }^{11}$ relatou um caso em região anterior de maxila em uma paciente pediátrica. Após a sua remoção, a análise macroscópica consistia em múltiplas estruturas dentiformes malformadas. Durante a análise histopatológica os cortes revelaram esmalte, 
dentina, cemento e tecidos semelhantes a polpa em algumas áreas. Essas estruturas estavam organizadas regularmente em algumas áreas, em outras, elas estavam dispostas de maneira desordenada, o que configurou características histológicas do tipo misto. ${ }^{11}$

Segundo a Organização Mundial da Saúde, ${ }^{3}$ essa neoplasia benigna tem prevalência mais comum entre os tumores odontogênicos. Em 2003, Cuesta e colaboradores ${ }^{15}$ relataram que sua prevalência pode chegar até $67 \%$, enquanto Guerrisi e colaboradores ${ }^{17}$ referiram, em crianças e adolescentes, $50,9 \%$ dos casos. Apesar do odontoma ser um dos tumores mais comuns, o tipo composto-complexo raramente é visto. No levantamento bibliográfico realizado para compor o estudo de Torul, ${ }^{11}$ em 2018, foram encontradas apenas cinco citações (quatro relatos de casos clínicos e um estudo retrospectivo) sobre a variante compostocomplexo na literatura de língua inglesa. ${ }^{11}$

\section{ReLATO do CAso}

Este relato de caso foi descrito de acordo com a avaliação de qualidade ancorada nas diretrizes do checklist CARE (case report guidelines). Uma jovem de 15 anos de idade, negra, procurou o Serviço de Cirurgia Oral e Maxilofacial do Hospital Federal dos Servidores do Estado do Rio de Janeiro, com queixa de aumento de volume em palato com evolução de aproximadamente dois anos, sem tratamento ou investigação prévia. Não apresentava histórico de trauma ou outras doenças de base relevantes. Ao exame clínico extraoral observou-se discreto aumento de volume em hemiface direita, elevando a asa do nariz e lábio superior ipsilaterais. $\mathrm{Na}$ avaliação intraoral, apresentava dentição permanente incompleta e em acompanhamento ortodôntico. Foi observado aumento expansivo em região palatina de maxila do lado direito próximo aos elementos dentários anteriores superiores, indolor, de consistência dura à palpação, medindo aproximadamente três centímetros de diâmetro. A mucosa intraoral adjacente estava íntegra e não apresentava alterações extraorais (FIGURA $1 \mathrm{~A}, \mathrm{~B}$ e C).

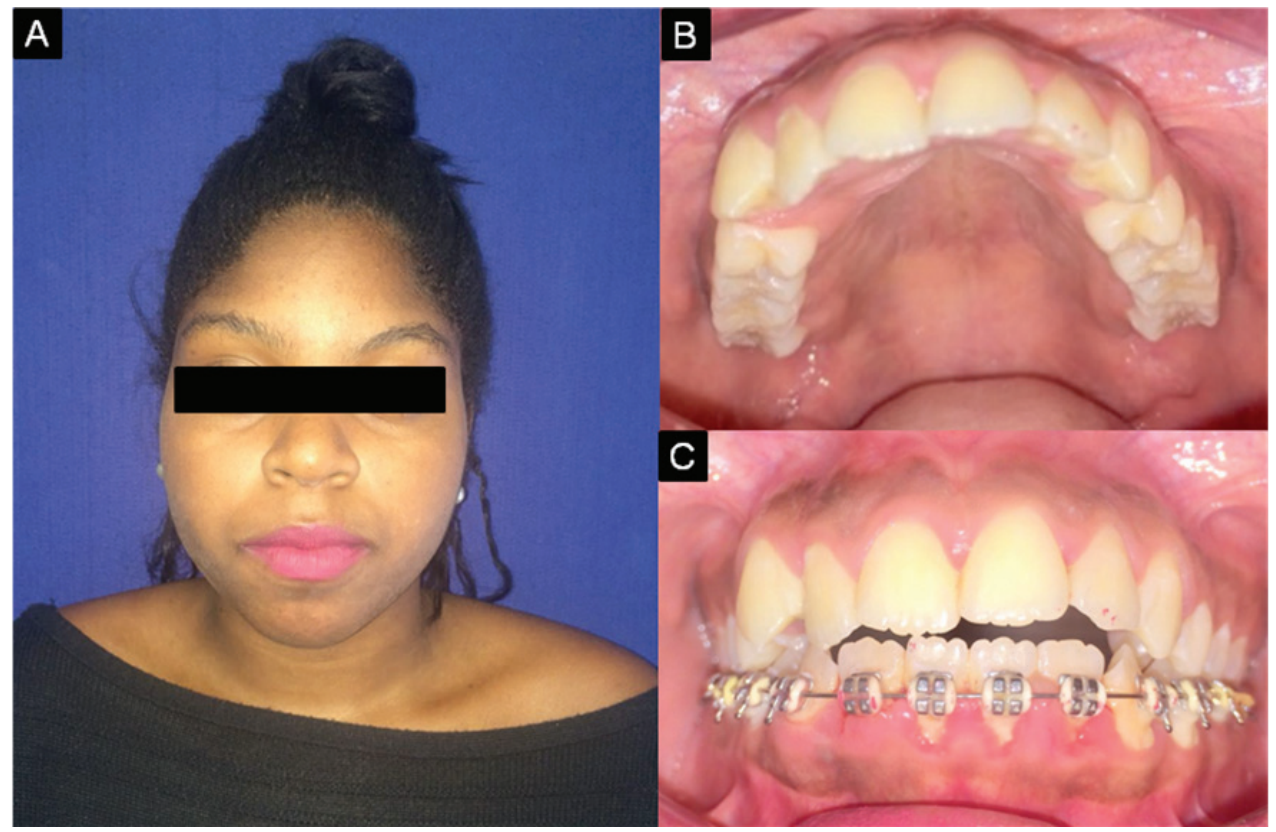

Figura 1: A. Vista extraoral frontal da paciente apresentando apagamento do filtro nasolabial, sugerindo aumento de volume. B. Vista intraoral da arcada superior demonstrando abaulamento vestibular e ligeiro abaulamento pela cortical palatina com afastamento do elemento dentário 13. C. Vista intraoral da paciente em oclusão, mostrando o mau posicionamento dos dentes no primeiro quadrante. 
Foi solicitada tomografia computadorizada de feixe cônico que demonstrou uma imagem semelhante a uma massa hiperdensa e mista, sem organização definida e delimitada por um halo hipodenso em toda sua extensão, acometendo a porção anterior de maxila direita, próximo as raízes dos dentes 11 a
13. Em outros cortes tomográficos também era possível observar, com maior precisão, pequenas e múltiplas imagens hiperdensas ou mistas semelhantes a estruturas dentárias que causavam deslocamento radicular (FIGURA 2 A, B e C).

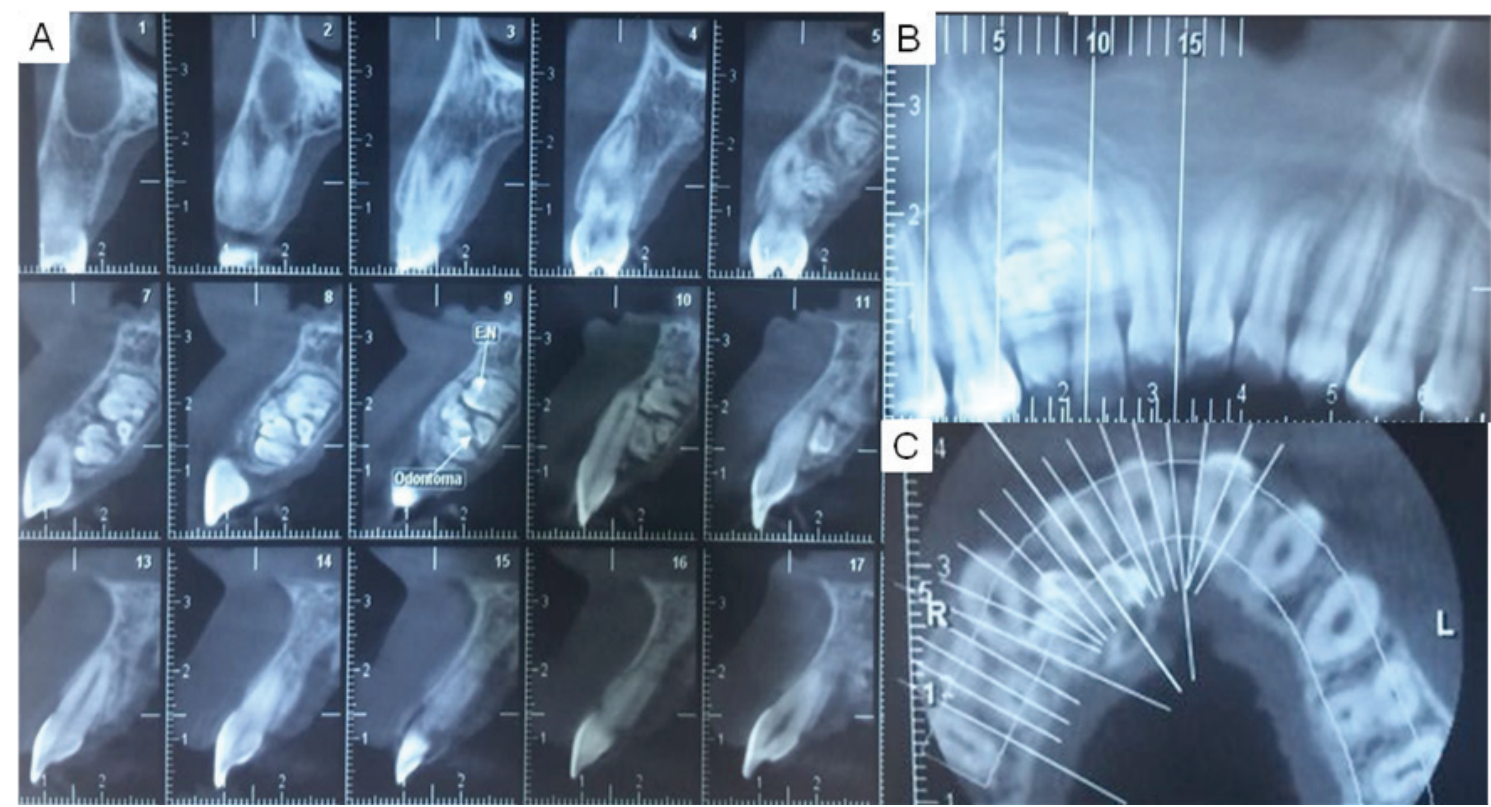

Figura 2: A. Corte sagital da tomografia computadorizada de feixe cônico com múltiplas estruturas de coloração mista provocando afastamento das raízes, sem reabsorção radicular, medindo aproximadamente $10 \mathrm{~cm}$. B. Corte coronal onde é possível observar a imagem radiopaca próxima aos ápices dos dentes envolvidos. C. Corte axial demonstrando relação entre as lesões com os elementos 11, 12 e 13.

Com base nos achados clínicos e dados radiográficos, a hipótese diagnóstica sugerida foi de odontoma complexo e o plano de tratamento proposto de excisão completa da lesão e análise histopatológica do material para confirmar o diagnóstico final. A presença de um halo radiolúcido ao redor da lesão permitiu um ponto de clivagem, o que favoreceu a exérese completa, sob anestesia local, através de uma incisão mucoperiosteal em região palatina de maxila. A remoção da lesão foi realizada com o uso de brocas rotatórias sem lesar estruturas anatômicas e dentárias adjacentes, permitindo um fechamento primário do retalho cirúrgico (Figura 3- A, B e C).
A peça cirúrgica constituída predominantemente de tecidos duros foi encaminhada para análise anatomopatológica. $\mathrm{Na}$ análise macroscópica foram recebidos oito fragmentos de tecido duro e um fragmento de tecido mole aderido ao fragmento maior de tecido duro. $\mathrm{Na}$ análise microscópica, foi observado fragmento de tecido conjuntivo fibroso capsular permeado por ilhas de epitélio odontogênico e tecido mineralizado constituído predominantemente por dentina, com focos de matriz de esmalte e polpa dentária, predominando áreas com formação de dentículos e outras áreas com depósito desorganizado. O laudo anatomopatológico foi de odontoma composto com áreas de complexo (Figura 4- A, B e C). 


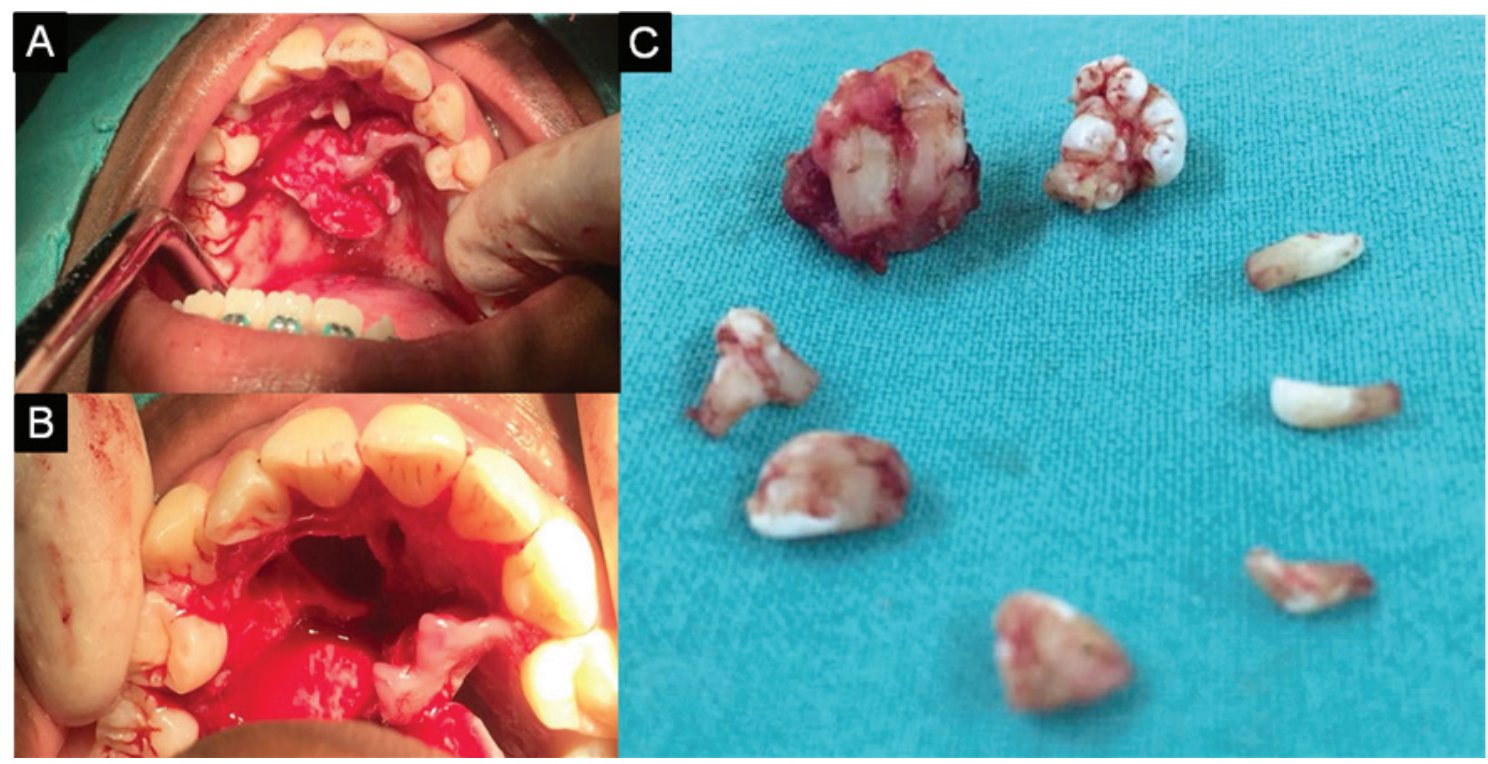

Figura 3: A. Incisão em região palatina anterior. B. Loja cirúrgica completa da lesão. C. Aspecto da peça cirúrgica: material semelhante a dentículos e uma massa maior deformada.

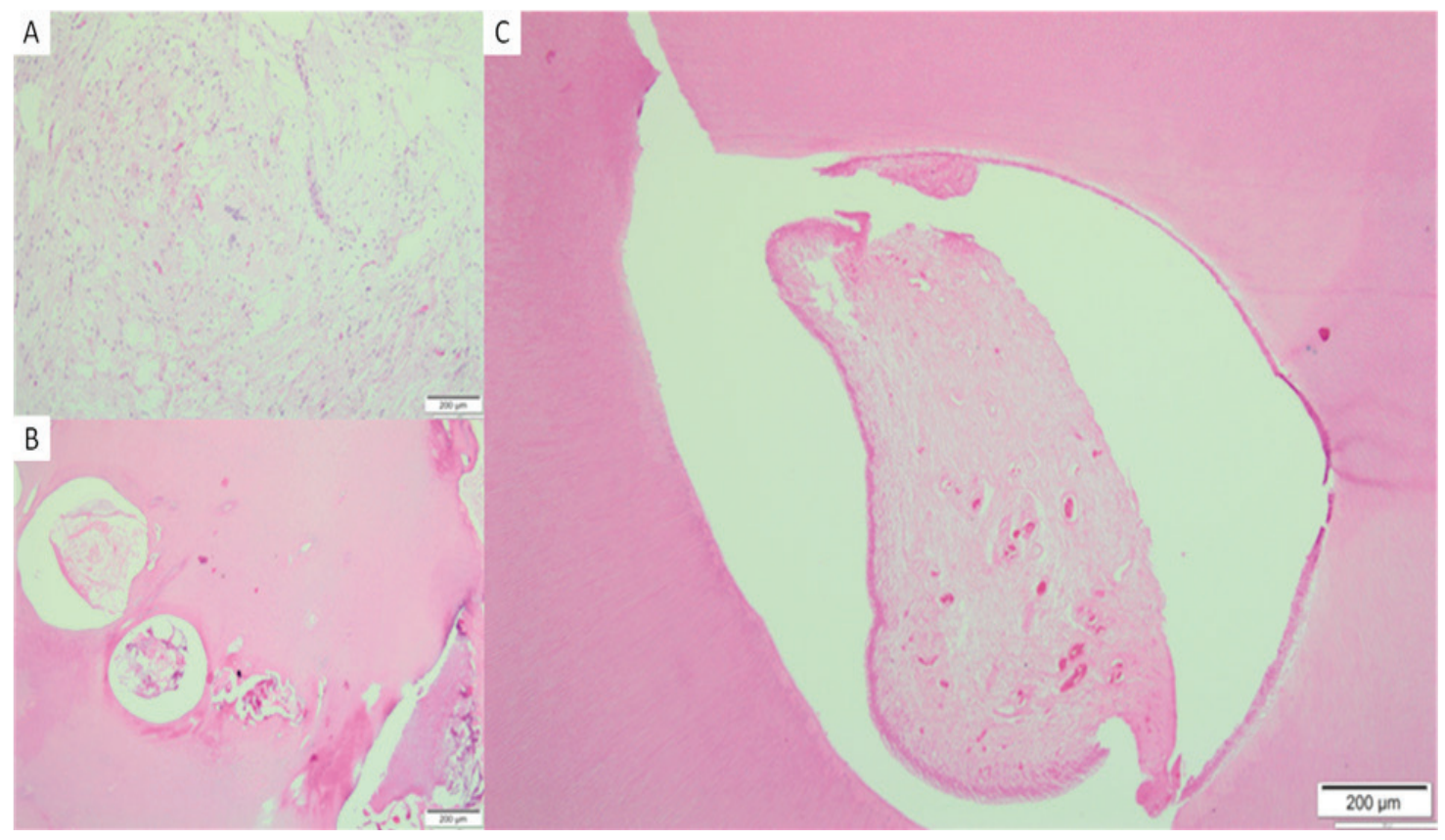

Figura 4: A. Fotomicrografias. Cápsula composta por tecido conjuntivo fibroso entremeado por ilhas de epitélio odontogênico (HE - 10X); B. Área de odontoma complexo exibindo esmalte, dentina e tecido conjuntivo semelhante a polpa dentária depositados de forma desorganizada (HE - 10x). C. Área de odontoma composto exibindo tecido dentinário e no centro tecido semelhante a polpa dentária com presença de odontoblastos na periferia e vasos sanguíneos em seu interior (HE - 10x). 
No pós-operatório, a paciente foi acompanhada inicialmente a cada sete dias e após, com intervalos de quinze dias, através de radiografias panorâmicas de controle. Neste momento encontra-se com 12 meses de pósoperatório da cirurgia com cicatrização óssea adequada e com um controle progressivo do processo de remodelação óssea.

\section{Discussão}

O diagnóstico desta lesão em questão se dá de maneira fácil, uma vez que possui características próprias no exame radiográfico. O odontoma composto aparece como várias estruturas, de forma e tamanho variáveis, envoltos por uma zona radiolúcida, sendo sua imagem patognomônica e semelhante morfologicamente a um elemento dentário, o que, muitas vezes, faz que o diagnóstico dessa lesão seja puramente clínico e radiográfico. Já o tipo complexo apresenta-se como massa radiopaca amorfa, envolvida também por uma estreita zona radiotransparente, formado por um padrão de distribuição desordenado. ${ }^{4,5}$ No caso supracitado, o diagnóstico incomum de odontoma complexo-composto trouxe características clínicas como aumento na região palatina da maxila e retenção de elementos permanentes, compatíveis com casos comuns desta lesão na literatura e em experiência clínica de nosso Serviço.

A maioria desses tumores é detectável a partir da segunda década de vida ${ }^{1,4}$ e por se tratar de uma lesão assintomática, muitas vezes só é descoberto através de exames radiográficos ou ao ser investigado o atraso da erupção de algum elemento dentário. ${ }^{4,5}$ Porém, Sedano e Pindborg ${ }^{1}$ identificaram pequenos odontomas entre raízes de dentes irrompidos sem associação com atraso na erupção. Os tipos divergem em relação a sua localização e aparecem em região anterior e posterior de ambos os maxilares, assim como, a variante composto-complexo parece não ter predileção por uma área especifica, Graydon ${ }^{10}$ apresentou um relato deste tipo na região de molares superiores, $\mathrm{Nóia}^{4}$ em região anterior de mandíbula e Torul, ${ }^{11}$ assim como o caso apresentado neste trabalho, em região anterior de maxila.

Uma série de lesões se assemelha radiograficamente a esses tumores e o diagnóstico diferencial deve ser discutido para eliminar hipóteses de diagnóstico que alterem o plano de tratamento. ${ }^{9}$ Quando o odontoma composto se apresenta na sua aparência clássica, estruturas radiopacas semelhantes a dentes no interior de zona radiolúcida, acaba gerando pouca divergência na hipótese diagnóstica e leva a um fácil diagnóstico. 1,4,6,10 $^{-1}$ Outras lesões como fibroma ossificante, tumor odontogênico adenomatoide, osteomas ou osteítes condensantes podem facilmente ser confundidas com os odontomas complexos e o diagnóstico pode ser realizado somente após $\mathrm{o}$ procedimento cirúrgico e análise histopatológica. ${ }^{9}$ No caso clínico relatado, a hipótese diagnóstica de odontoma complexo foi ancorada nos achados clínicos e radiográficos mais relevantes da lesão, de uma imagem radiopaca homogênea desorganizada e amorfa, sendo o diagnóstico final posteriormente complementado pela associação do tipo composto-complexo, após o exame histopatológico.

A excisão cirúrgica total é a opção de escolha como forma de tratamento para essa alteração intraóssea e está de acordo com as condutas terapêuticas sugeridas. Esse tipo de tratamento cirúrgico pode ser considerado conservador, uma vez que a remoção desse tumor se dá de maneira fácil devido à presença da área de clivagem entre a lesão e o osso. Existe um consenso na literatura em relação à presença de dentes associados a essas lesões e, em muitos casos, o dente que pode estar impactado apresenta potencial para erupcionar 
normalmente após a remoção da lesão. Existem trabalhos que também destacam a dificuldade na remoção da lesão sem lesar os dentes adjacentes, assim o dente impactado também deve ser removido no momento da cirurgia por não apresentar um bom potencial para erupcionar e chegar à arcada dentária. 1, 2, 4, 6, 11

A remodelação óssea que ocorre após a remoção completa dessa lesão é observada na maioria dos casos, o que favorece e confirma que as chances de recidiva são remotas. .,9,14 $^{2}$ Neste trabalho, após 12 meses de acompanhamento pós-operatório não foram observadas complicações ou recidivas, comprovando o prognóstico bastante favorável, o baixo índice de recidiva dessa lesão e o sucesso do correto plano de tratamento realizado.

\section{CONSIDERAÇÕES FINAIS}

O diagnóstico final do odontoma é feito pela associação dos achados clínicoradiográficos e histopatológicos. Seu tratamento demanda um planejamento preciso, evitando possíveis lesões às estruturas nobres próximas. Este relato destaca-se pelo diagnóstico incomum devido às características histopatológicas simultâneas do tipo compostocomplexo.

\section{REFERÊNCIAS}

1. Sedano HO, Pindborg JJ. Ghost cell epithelium in odontomas. J Oral Pathol 1975; 4:27-30. [https://doi.org/10.1111/j.1600-0714.1975. tb01737.x]

2. Silva DJS, Souza JJ, Almeida RAC, Andrade ESS. Estudo clínico-patológico de odontomas diagnosticados no Laboratório de Patologia Bucal da Faculdade de Odontologia de Pernambuco-FOP. Rev Cir Traumatol Buco-Maxilo-Fac 2015; 15 (4):31-36. [http:// revodonto.bvsalud.org/scielo.php?script=sci_ abstract\&pid=S1808-52102015000400005\&Ing =pt\&nrm=iso\&tlng=PT]
3. El-Naggar AK, Chan JKC, Grandis JR, Takata T., Slootweg PJ. WHO classification of head and neck tumours. WHO/IARC Classification of Tumours, 4th Ed. Lyon: IARC Press; 2017.

4. Nóia CF et al. Odontoma composto-complexo: Relato de caso. UFES Rev Odontol 2008; 10 (4):59-63. [https://doi.org/10.21722/rbps. v0i0.460]

5. Santos MESM, Silva ARBL, Florêncio AG, Silva UH. Odontoma como fator de retenção dentária: Relato de casos clínicos. Rev Cir Traumatol Buco-Maxilo-Fac 2010; 10 (2):25-30. [http:// revodonto.bvsalud.org/scielo.php?script=sci_ar ttext\&pid=S1808-52102010000200005]

6. Freitas DA, Freitas VA, Mol VC, Neto LM, Mol VC. Elemento dental impactado por odontoma composto. Rev Bras Cir Cabeça Pescoço 2009; 38 (3):198-199. [http://www.sbccp.org.br/ wp-content/uploads/2014/11/art_152.pdf]

7. Cé PS, Prazeres C, Santos FL, Woltmann M. Odontoma complexo: Relato de caso clínico atípico. RFO 2009; 14 (1):56-60. [http:// download.upf.br/editora/revistas/rfo/1401/56_60.pdf]

8. Bordini Jr J, Contar CM, Sarot JR, Fernandes A., Machado MAN. Multiple compound odontomas in the jaw: Case report and analysis of the literature. J Oral Maxillofac Surg 2008; 66 (12):2617-2620. [https://doi.org/10.1016/j. joms.2007.08.027]

9. Soluk MT, Pehlivan S, Olgac V, Aksakalli N, Alatli C. Clinical and histopathological investigation of odontomas: Review of the literature and presentation of 160 cases. J Oral Maxillofac Surg 2012; 70 (6):1358-1361. [https://www. ncbi.nlm.nih.gov/pubmed/21840103]

10. Smith GC. An interesting presentation of a complex-compound odontome. Aust Dent J 1985; 30 (4):265-267. [https://www.ncbi.nlm. nih.gov/pubmed/3866527]

11. Torul D, Keskin M, Gun S, Odabasi D. Complex-compound odontoma: A rare clinical presentation. ODOVTOS - Int J Dent Sc 2018; 1:41-44. [https://revistas.ucr.ac.cr/index.php/ Odontos/article/view/33920]

12. Rosoff ML. Odontoma. Am J Orthodontics and Oral Surg (Oral Surg Sect) 1943; 29: 332-340. [https://doi.org/10.1016/S0096-6347(43)90406-5] 
13. Tomizawa M, Otsuka Y, Noda T. Clinical observations of odontomas in japanese children: 39 cases including one recurrent case. Int J Paediatr Dent 2005; 15 (1):37-43. [https:// www.ncbi.nlm.nih.gov/pubmed/15663443]

14. Ali Azhar D, Kota MZ, El-Nagdy S. An unusual erupted complex composite odontoma: A rare case. Case Rep Dent 2013; 2013:106019. [https://www.ncbi.nlm.nih.gov/ pubmed/23346425]

15. Veis A, Tziafas D, Lambrianidis T. A case report of a compound odontoma causing delayed eruption of a central maxillary incisor: Clinical and microscopic evaluation. J Endod 2000; 26:477479. [http://dx.doi.org/10.1097/00004770200008000-00012]

16. Philipsen HP, Reichart PA. Classification of odontogenic tumours: A historical review. J Oral Pathol Med 2006; 35: 525-9. [https://doi. org/10.1111/j.1600-0714.2006.00470.x]

17. Castellon CG, Valenzuela JSP, Lira LA. Tumores Benignos dos Maxilares, I. Radiologia Odontológica. 5th ed. São Paulo (SP): Artes Médicas; 2000. p. 507-532.

18. Cuesta SA, Albiol JG, Aytés LB, Escoda CG. Revisión de 61 casos de odontoma. Presentación de um odontoma complejo erupcionado. Med Oral 2003; 8:366-373. [http:// www.medicinaoral.com/pubmed/medoralv8_i5_ p366.pdf]

19. Guerrise M, Pilone M, Keszler A. Odontogenic tumors in children and adolescents. A 15-year retrospective study in Argentina. Med Oral Patol Oral Cir Bucal 2007; 5 (12):180-185. [https:// www.ncbi.nlm.nih.gov/pubmed/17468710]

20. Thoma KH, Goldman HM. Odontogenic tumors: A classification based on observations of epithelial, mesenchymal and mixed varieties. Am J Pathol 1946; 22: 433-71. [https://www. ncbi.nlm.nih.gov/pubmed/19970874]

21. Wanjari SP, Tekade SA, Parwani RN, Managutti SA. Dentigerous cyst associated with multiple complex composite odontomas. Contemp Clin Dent 2011; 2 (3):215-217. [https://doi. org/10.4103/0976-237X.86465]

22. Miles AE. A cystic complex composite odontome. Proc R Soc Med 1951; 44 (1):5155. [https://www.ncbi.nlm.nih.gov/pmc/articles/ PMC2081599/].

Submetido em: 22-4-2020

Aceito em: 14-9-2020 